\title{
Adherencia al tratamiento de osteoporosis en pacientes posmenopáusicas en un hospital de referencia, Perú 2013
} Osteoporosis medication adherence in postmenopausal women in a referral hospital, Peru 2013

\author{
Paul Jesús Tejada-Llacsa ${ }^{1,2}$, Judith Cahuana-Aparco ${ }^{1,2}$, Joel Chalco Huaman ${ }^{1,3}$, \\ Catharine De Freitas Vidal ${ }^{1,3}$, Jhon Eduardo Cabello Leon ${ }^{4}$ \\ Facultad de Medicina Humana, Universidad Nacional Mayor de San Marcos (UNMSM), Lima, Perú. \\ ${ }^{2}$ Asociación para el Desarrollo de la Investigación Estudiantil en Ciencias de la Salud (ADIECS), Lima, Perú. \\ ${ }^{3}$ Sociedad Científica de San Fernando (SCSF), Lima, Perú. \\ ${ }^{4}$ Servicio de Reumatología del Hospital Nacional Alberto Sabogal Sologuren, EsSalud, Callao, Perú.
}

\begin{abstract}
Resumen
Introducción: A pesar de la eficacia del tratamiento para la osteoporosis, alrededor de $50 \%$ de las pacientes tienden a descontinuarlo. Objetivos: Evaluar la frecuencia de la baja adherencia al tratamiento de la osteoporosis y sus características asociadas. Diseño: Estudio descriptivo, transversal y observacional. Lugar: Servicio de Reumatologia, Hospital Nacional Alberto Sabogal, Callao, Perú. Participantes: Pacientes posmenopáusicas con diagnóstico de osteoporosis. Intervenciones: En febrero y marzo 2013 se aplicó un cuestionario de 32 preguntas a mujeres posmenopáusicas con diagnóstico de osteoporosis. Se usó la prueba de Morisky-Green (MG) para medir la adherencia al tratamiento. Principales medidas de resultados: Porcentaje y caracteristicas asociadas a la no adherencia. Resultados: Se encuestó a 81 pacientes; la edad media fue 67,1 años $( \pm$ 8,2); el porcentaje de no adherencia al tratamiento fue $80,2 \%$. Las características asociadas fueron número de medicamentos adicionales, numero de comorbilidades, diabetes y la falta de búsqueda de información sobre la enfermedad. Conclusiones: Existe un elevado porcentaje de no adherencia a la osteoporosis, por lo que sugerimos realizar futuros estudios para caracterizar este problema en nuestro contexto.
\end{abstract}

Palabras clave: Osteoporosis, adhesión, tratamiento.

\section{Abstract}

Background: About $50 \%$ of osteoporotic patients tend to discontinue treatment even though its effectiveness in lowering fracture risk has been largely proven. Objective: To assess non-adherence to osteoporosis treatment and its associated factors. Design: Cross-sectional study. Participants: Osteoporotic postmenopausal women. Setting: Rheumatology service, Alberto Sabogal Hospital, EsSalud, Callao, Peru. Interventions: Osteoporotic postmenopausal women were invited to complete a questionnaire. Morisky-Green (MG) test was used to measure adherence. Main outcome measures: Percentage and associated characteristics to non-adherence. Results: Eighty-one women participated in the questionnaire, the mean age was 67.1 years $( \pm 8.2)$, and the percentage of non-adherence to treatment was $80.2 \%$. Number of comorbidities, number of additional drugs, diabetes mellitus and lack of interest in search of information on the disease were associated to non-adherence. Interpretation: Percentage of non-adherence was high, in contrast to other studies. Future studies to characterize this problem in our context are needed.

Keywords: Osteoporosis, medication adherence, treatment.

An Fac med. 2015;76(1):43-6 / doi:10.15381/anales.v76i1.11074

\section{INTRODUCCIÓN}

La osteoporosis es una enfermedad sistémica del esqueleto óseo caracterizada por una pérdida de masa ósea y un deterioro de la microarquitectura, que predispone a las fracturas por fragilidad ${ }^{(1)}$. Debido a que es asintomática, el paciente no percibe su enfermedad y resta importancia a su tratamiento ${ }^{(2)}$.

Actualmente, la osteoporosis afecta aproximadamente 200 millones de mujeres en todo el mundo, cifra que se ve en aumento. Es así que para el año
2020, en EE UU habrá aproximadamente 14 millones de adultos mayores con el diagnóstico de osteoporosis ${ }^{(3)}$.

Las fracturas por fragilidad debida a osteoporosis disminuyen la calidad de vida, aumentan los costos en el tratamiento y la mortalidad. Se estimó que, en la población hispana, en 2025 se duplicará la incidencia de fracturas y los costos asociados ${ }^{(4)}$. En el Perú, la mortalidad después del primer año de una fractura ha sido encontrada en $16,5 \%{ }^{(5)}$.
El tratamiento para la osteoporosis ha demostrado ser efectivo en la disminución de la tasa de fracturas; no obstante, es necesario seguir un tratamiento prolongado ${ }^{(6)}$. Se ha observado que un promedio de $50 \%$ de los pacientes tiende a descontinuar el tratamiento ${ }^{(7)}$.

Se describe que los factores asociados a la pobre adherencia son: 1) El medicamento: vía de administración, frecuencia de dosis tomada, efectos adversos; 2) El paciente: conocimiento de las consecuencias de la enfermedad, 
creencias, comorbilidades; 3) Otros factores: mala relación médico paciente, la especialidad del médico y el soporte social ${ }^{(8,9)}$.

En el Perú, hasta donde se tiene conocimiento, no existen estudios que aborden este problema. Nuestro objetivo fue determinar la frecuencia de la baja adherencia al tratamiento para la osteoporosis y sus características asociadas, en las pacientes posmenopaúsicas atendidas en un consultorio externo de Reumatología.

\section{MÉTODOS}

Diseño descriptivo, transversal y observacional. La población de estudio comprendió a las pacientes posmenopaúsicas con diagnóstico de osteoporosis atendidas entre el 4 de febrero y el 30 de marzo del 2013 en el servicio de Reumatología del Hospital Nacional Alberto Sabogal Sologuren, EsSalud, Callao, Perú. No hubo muestreo, se trabajó con la población comprendida en dicho periodo de manera consecutiva, según el horario de consulta.

Incluimos a las pacientes mayores de 45 años, diagnosticadas de osteoporosis por densitometría mineral ósea (DMO) y que eran tratadas con calcio $(500 \mathrm{mg}$ dos veces al día), vitamina D (ergocalciferol 600000 UI mensual) y alendronato (70 mg semanal). Se excluyó a las pacientes que vivían en casa de retiro o que estuvieran participando en algún ensayo clínico

Nuestra principal variable fue la adherencia al tratamiento. Consideramos a la adherencia como la aproximación que tiene la conducta del paciente en relación a su medicación prescrita (alendronato + calcio + vitamina D) ${ }^{(10)}$. Para medirla usamos la prueba de Morisky-Green, que consiste en cuatro preguntas y califica a la adherencia en relación al número de respuestas correctas: 4 respuestas correctas, 'buen cumplidor con alta adherencia al tratamiento'; 2 o 3 respuestas correctas, 'cumplidor medio con adherencia media'; 1 o ninguna respuesta correcta, 'baja adherencia al tratamiento'. Para el análisis se consideró como adherente si respondía bien las 4 preguntas y no adherente si tuvo alguna respuesta incorrecta $^{(2)}$.

Evaluamos variables sociodemográficas, como edad, nivel de educación y si vivía sola. Además, año de diagnóstico de la osteoporosis, si tuvo fracturas y la localización de estas, comorbilidades, el número de pastillas adicionales que toma la paciente además de su medicación para la osteoporosis, el nivel de conocimientos que tiene en relación a la osteoporosis, la actitud hacia la osteoporosis y preguntas acerca de la atención en consulta.

El conocimiento acerca de la osteoporosis fue evaluado mediante la prueba de Batalla ${ }^{(11)}$, que mide el conocimiento en base a 3 preguntas, obteniendo los siguientes resultados:

a. Aceptable: afirma que la osteoporosis es una enfermedad crónica, pero puede prevenirse con medicación y/o cambios en el estilo de vida. Además, conoce al menos una complicación de la enfermedad.

b. Inaceptable: el paciente no conoce al menos una complicación de la osteoporosis.

c. Medianamente aceptable o moderado: cualquier respuesta intermedia.

La actitud frente a la enfermedad se midió en respuesta en escala de Likert (muy de acuerdo, de acuerdo, indiferente, desacuerdo, muy en desacuerdo), en relación a: 1) Me preocupa tener osteoporosis. 2) La medicación para la osteoporosis es buena para mí. 3) La medicación para la osteoporosis me mantiene activo. Además, se realizó una pregunta con respuesta Sí/No: ¿Ha buscado en otras fuentes de información (internet, periódico, revistas o libros) acerca de la osteoporosis?

Las preguntas acerca de la atención en la consulta fueron: iSu doctor le explicó acerca de su enfermedad? iSiente que fue demasiado rápido el tiempo en que le explicó?
Considerando el principio bioético de autonomía, se utilizó un consentimiento informado antes de aplicar la encuesta a cada paciente; después, se le explicó acerca de su enfermedad. Las encuestas fueron archivadas de forma anónima respetando la confidencialidad de la información. Se realizó el trabajo contando con la aprobación del comité de ética del Hospital Nacional Alberto Sabogal Sologuren (HNASS).

El análisis estadístico consistió en una primera etapa de análisis descriptivo, por el cual se obtuvo medidas de resumen y distribuciones de frecuencia, con un intervalo de confianza de $95 \%$. En la segunda etapa se hizo el análisis bivariado, que consistió en la determinación de la asociación con los resultados obtenidos por la prueba de Morisky-Green. Se calculó como medida de fuerza de asociación el odds ratio (OR) con intervalos de confianza de 95\% (IC 95\%), con un nivel de significación de 0,05. El análisis de los datos se realizó con el programa Stata versión 12.

\section{RESULTADOS}

Se consideró un total de 113 pacientes, de las cuales 15 no aceptaron la entrevista y 17 encuestas fueron mal llenadas, quedando 81 pacientes mujeres posmenopáusicas en el estudio. La media de la edad fue 67,1 $\pm 8,2$ años. La media del tiempo de diagnóstico de osteoporosis fue 6,5 \pm 5 años. La comorbilidad más frecuente fue la hipertensión arterial, en $44,4 \%$. Las variables demográficas, clínicas y las comorbilidades se especifican en la tabla 1.

Mediante la prueba de MoriskyGreen se encontró 19,8\% de pacientes con buena adherencia y $80,2 \%$ sin buena adherencia (adherencia baja 18,5\% y adherencia media 61,7\%).

En relación al conocimiento de la enfermedad, según la prueba de Batalla $65,4 \%$ de las pacientes mostró un nivel de conocimiento inaceptable; por otro lado, los niveles de conocimiento medianamente aceptable y aceptable representaron $17,3 \%$ para ambos casos. 
Tabla 1. Características de la población estudiada de mujeres posmenopáusicas con tratamiento para la osteoporosis.

\begin{tabular}{|c|c|}
\hline Características & Valor/Porcentaje \\
\hline \multicolumn{2}{|l|}{ Características sociodemográficas } \\
\hline - Edad (años) & $67,1 \pm 8,2$ \\
\hline \multicolumn{2}{|l|}{ - Nivel de educación } \\
\hline Ninguno & 7,4 \\
\hline Primaria & 42,0 \\
\hline Secundaria & 30,9 \\
\hline Superior & 19,8 \\
\hline - Vive solo (sí) & 22,2 \\
\hline \multicolumn{2}{|l|}{ Características clínicas densitométricas } \\
\hline - Años desde el diagnóstico ( $\bar{x} \pm D E)$ & $6,5 \pm 5$ \\
\hline - Pacientes con por lo menos una fractura & 49,4 \\
\hline - Tiempo desde la última fractura (años) & $3,8 \pm 4,5$ \\
\hline \multicolumn{2}{|l|}{$\begin{array}{l}\text { - Pacientes con por lo menos una fractura de acuerdo a } \\
\text { localización }\end{array}$} \\
\hline Antebrazo & 30,8 \\
\hline Codo & 5,1 \\
\hline Cadera & 30,8 \\
\hline Otros & 33,3 \\
\hline \multicolumn{2}{|l|}{ Comorbilidades asociadas } \\
\hline - Hipertensión & 44,4 \\
\hline - Dislipidemia & 27,2 \\
\hline - Artritis reumatoide & 13,6 \\
\hline - Diabetes & 17,3 \\
\hline - Enfermedad del corazón & 12,3 \\
\hline - Accidente cerebro vascular & 6,2 \\
\hline - Otros & 27,2 \\
\hline
\end{tabular}

No se observó asociación entre conocimientos y la adherencia al tratamiento.

Dentro del ítem de número de medicamentos adicionales, se observó que $21 \%$ tomaba solo 1 pastilla adicional y $79 \%$ más de dos pastillas (58\% de 2 a 5 pastillas y $21 \%$ más de 6 pastillas al día).

En relación a la actitud frente a la enfermedad, $82,7 \%$ refirió estar preocupada por su enfermedad, $71,6 \%$ creía que el medicamento le hacía bien y $64,2 \%$ sentía que la mantenía activa. Además, 64,2\% refirió haber buscado información sobre la osteoporosis en otras fuentes (revistas, internet, libros o periódicos).
En cuanto al momento de la consulta, $61,7 \%$ sintió que la consulta fue demasiado rápida, y en solo $44,4 \%$ su doctor le explicó su enfermedad. No se encontró asociación de estos factores con la baja adherencia. Green.
Además, se realizó preguntas acerca de las creencias en relación a la osteoporosis; $25,9 \%$ creía que la osteoporosis era un cáncer del hueso y $64,2 \%$ afirmó que esta enfermedad causaba demasiado dolor.

Los resultados de la asociación entre los factores estudiados y la no adherencia según la prueba de Morisky-Green son descritos en la tabla 2.

\section{DISCUSIÓN}

El porcentaje de pacientes con falta de adherencia en nuestro estudio $(80,2 \%)$ es mayor en comparación con estudios realizados en España, donde al aplicar el mismo instrumento para la adherencia al tratamiento de calcio, vitamina D y bisfosfonatos, se encontró una baja adherencia entre 44 y $68 \%{ }^{(2,12)}$.

Hallamos asociación entre la baja adherencia tanto con el número de comorbilidades y el número de fármacos adicionales que tomaba el paciente (dos o más fármacos); estos dos factores pueden haber aumentado debido al grupo etario en el que se desarrolla esta enfermedad, así como a la presencia de enfermedades como diabetes e hipertensión, las cuales tiene un alto porcentaje de no adherencia ${ }^{(13)}$. Contrariamente a nuestros hallazgos en relación al número de fármacos adicionales, en algunos estudios no se encuentra asociación; inclusive, los que tienen una mayor cantidad de fármacos adicionales tienen una mejor adherencia ${ }^{(14)}$.

Tabla 2. Factores asociados a la baja adherencia al tratamiento según la prueba de Morisky

\begin{tabular}{|c|c|c|c|c|}
\hline Variable & Categoría & $\mathrm{OR}$ & IC (95\%) & Valor $P$ \\
\hline Diabetes & Sí / No & 1,3 & 1,1 a 1,5 & 0.04 \\
\hline $\begin{array}{l}\text { Búsqueda de información (en } \\
\text { periódicos, libros, internet) sobre } \\
\text { la enfermedad }\end{array}$ & No / Sí & 4,0 & 1,3 a 12,7 & 0,01 \\
\hline $\begin{array}{l}\text { Número de medicamentos } \\
\text { adicionales }\end{array}$ & Más de uno / solo 1 & 6,2 & 1,3 a 14,1 & 0,01 \\
\hline Número de comorbilidades & Más de uno / ninguna & 5,4 & 1,3 a 22 & 0,01 \\
\hline
\end{tabular}


En relación al conocimiento de la enfermedad, se halló un porcentaje de conocimiento inaceptable de 65,4\%, similar al de otras publicaciones donde varía entre 50 y $70 \%$ de conocimiento inaceptable ${ }^{(11)}$. En nuestro estudio, al igual que otros, se ha visto que el grado de conocimiento no estuvo asociado con la baja adherencia ${ }^{(11,14,15)}$.

Encontramos un alto porcentaje de pacientes que refirió preocuparse por su enfermedad y que percibía que la medicación le hacía bien. Sin embargo, no hubo asociación de estos factores con una buena adherencia, a diferencia de lo que se publicado en la literatura ${ }^{(16)}$. No obstante, hubo asociación entre las que no buscaban información acerca de la osteoporosis y la baja adherencia, lo cual es comparable con otros estudios ${ }^{(9)}$. Sin embargo, en nuestra población $49,4 \%$ de las pacientes tuvo como grado de educación hasta nivel primaria, lo que puede influir en los resulta$\operatorname{dos}{ }^{(9,17)}$.

La alta percepción de una atención rápida y sin recibir una explicación acerca de su enfermedad no resultó asociarse a una baja adherencia. Además, hubo un alto porcentaje de pacientes que estaban seguras que la osteoporosis causaba dolor y que es un 'tipo de cáncer del hueso'. Si bien es cierto no encontramos asociación estadística de estos factores y una baja adherencia, está descrito que una relación médico paciente en el cual exista una buena empatía, haya una explicación acerca de la enfermedad y se aborde el significado del valor de la densidad de masa ósea, lo que resulta beneficioso para una mejor adherencia al tratamiento ${ }^{(16)}$.

Dentro de las limitaciones de nuestro estudio tenemos que considerar el bajo número de participantes. Por otro lado, es importante considerar que medir la adherencia a algún tratamiento constituye un reto. Es así que existen varias formas de medir la adherencia. En este estudio se utilizó la prueba de
Morisky-Green, que tiene la ventaja de ser fácilmente aplicable en la clínica (2). Sin embargo, sobreestima la baja adherencia. A pesar de estas dificultades, este método resulta útil para darnos una idea de la magnitud del problema ${ }^{(15,18)}$. Además, es necesario acotar que la adherencia al tratamiento varía de acuerdo al medicamento y la frecuencia de administración. En nuestro estudio todas las pacientes usaron alendronato semanalmente, el cual se ha visto tiene una mejor adherencia de $62,5 \%{ }^{(18)}$.

Se concluye que existe un alto porcentaje de pacientes que no son adherentes al tratamiento de la osteoporosis, por lo que se sugiere realizar futuros estudios para caracterizar este problema en nuestro contexto.

\section{REFERENCIAS BIBLIOGRÁFICAS}

1. Guideline Committee DVO. DVO Guideline 2009 for Prevention, Diagnosis and Therapy of Osteoporosis in Adults. Osteologie. 2011;20:55-74.

2. Ros I, Guañabens N, Codina C, Peris P, Roca M, Monegal $A$, et al. Análisis preliminar de la adherencia al tratamiento de la osteoporosis. Comparación de distintos métodos de evaluación. Reemo. 2002;11(3):92-6.

3. Lane NE. Epidemiology, etiology, and diagnosis of osteoporosis. Am J Obstet Gynecol. 2006;194:S3-11.

4. Burge R, Dawson-hughes $B$, Solomon DH, Wong JB, King A, Tosteson A. Incidence and economic burden of osteoporosis-related fractures in the United States, 2005-2025. J Bone Miner Res. 2007;22(3):465-75.

5. Miraval T, Becerra F, Segami I. Fractura de cadera a trauma mínimo en mayores de 50 años: morbimortalidad y pronóstico funcional. Rev Peru Reumatol. 2001;7(2):7-16.

6. Cranney A, Wells G, Willan A, Griffith L, Zytaruk N, Robinson V, et al. II. Meta-analysis of alendronate for the treatment of postmenopausal women. Endocr Rev. 2002 Aug 1;23(4):508-16.

7. Ziller V, Kostev K, Kyvernitakis I, Boeckhoff J, Hadji P. Persistence and compliance of medications used in the treatment of osteoporosis - analysis using a large scale, representative, longitudinal German database. Int J Clin Pharmacol Ther. 2012 Mar 2;50(5):315-22.

8. Iversen MD, Vora RR, Servi A, Solomon DH. Factors affecting adherence to osteoporosis medications: a focus group approach examining viewpoints of patients and providers. J Geriatr Phys Ther. 2011 Apr-Jun;34(2):72-81. doi: 10.1097/ JPT.0b013e3181ff03b4.
9. Cortet B, Bénichou O. Adherence, persistence, concordance: do we provide optimal management to our patients with osteoporosis? Joint Bone Spine. 2006 Oct; 73(5):e1-7.

10. Cramer JA, Roy A, Burrell A, Fairchild CJ, Fuldeore MJ, Ollendorf DA, et al. Medication compliance and persistence : terminology and definitions. Value Heal. 2008;11(1):44-7.

11. Graña G, Miguélez R, Beaumont GH. Grado de conocimiento de la osteoporosis en mujeres posmenopausicas. REEMO. 2008;17(4):71-5.

12. Carbonell Abella C, Guañabens Gay N, Regadera Anechina L, Marín Rives JA, Taverna Llauradó E, Ayechu Redin MP; ADHEPOR. [Analysis of therapeutic compliance in women with osteoporosis]. Reumatol Clin. 2011 Sep-Oct;7(5):299-304. doi: 10.1016/j.reuma.2010.12.003.

13. Rolnick SJ, Pawloski P a, Hedblom BD, Asche SE, Bruzek RJ. Patient characteristics associated with medication adherence. Clin Med Res. 2013 Jun;11(2):54-65. doi: 10.3121/cmr.2013.1113.

14. Vytrisalova M, Blazkova S, Palicka V, Vlcek J, Cejkova M, Hala T, et al. Self-reported compliance with osteoporosis medication-qualitative aspects and correlates. Maturitas. 2008;60(3-4):223-9.

15. Sanfelix-Genoves J, Gil-Guillen VF, Orozco-Beltran D, Giner-Ruiz V, Pertusa-Martinez S, Reig-Moya $B$, et al. Determinant factors of osteoporosis patients' reported therapeutic adherence to calcium and / or vitamin D supplements. Drugs Aging. 2009;26(10):861-70. doi: 10.2165/11317070000000000-00000.

16. Warriner AH, Curtis JR. Adherence to osteoporosis treatments: room for improvement. Curr Opin Rheumatol. 2009 Jul;21(4):356-62. doi: 10.1097/ BOR.0b013e32832c6aa4.

17. Huas D, Debiais F, Blotman F, Cortet B, Mercier $F$, Rousseaux C, et al. Compliance and treatment satisfaction of post menopausal women treated for osteoporosis. Compliance with osteoporosis treatment. BMC Womens Health. 2010 Aug 20;10(26). doi: 10.1186/1472-6874-10-26.

18. Downey TW, Foltz SH, Boccuzzi SJ, Omar M a, Kahler KH. Adherence and persistence associated with the pharmacologic treatment of osteoporosis in a managed care setting. South Med J. 2006 Jun;99(6):570-5.

Artículo recibido el 13 de julio de 2014 y aceptado para publicación el 23 de octubre de 2014.

Declaración de conflictos de interés: Los autores declaran no tener conflictos de interés.

Fuente de financiamiento: Recursos propios.

Autor para correspondencia:

Paul Jesús Tejada Llacsa

Av. Gonzales Prada 719, Comas, Lima, Perú

Teléfono: 997851669

Correo electronico: paultejada24@gmail.com 